\title{
Submesoscale eddies in the South China Sea
}

\author{
Qinbiao $\mathrm{Ni}^{1}$, Xiaoming Zhai ${ }^{2}$, Chris Wilson ${ }^{3}$, Changlin Chen ${ }^{4}$, and Dake Chen ${ }^{1}$ \\ ${ }^{1}$ State Key Laboratory of Satellite Ocean Environment Dynamics, Second Institute of \\ Oceanography, Ministry of Natural Resources, Hangzhou, China \\ ${ }^{2}$ Centre for Ocean and Atmospheric Sciences, School of Environmental Sciences, \\ University of East Anglia, Norwich, UK \\ ${ }^{3}$ National Oceanography Centre, Liverpool, UK \\ ${ }^{4}$ Department of Atmospheric and Oceanic Sciences \& Institute of Atmospheric \\ Sciences, Fudan University, Shanghai, China
}

Corresponding author: Qinbiao Ni (niqinbiao@outlook.com)

\section{Key Points}

- Submesoscale eddies are detected automatically from ocean colour data and are analyzed statistically in the SCS

- The surface structure of submesoscale eddies shows the classical 'cat's-eye' pattern

- Submesoscale eddies can significantly modulate surface tracer distribution

This article has been accepted for publication and undergone full peer review but has not been through the copyediting, typesetting, pagination and proofreading process, which may lead to differences between this version and the Version of Record. Please cite this article as doi: 10.1029/2020GL091555.

This article is protected by copyright. All rights reserved. 


\begin{abstract}
Submesoscale eddies are often seen in high-resolution satellite-derived ocean colour images. To efficiently identify these eddies from surface chlorophyll data, here we develop an automatic submesoscale eddy detection method and apply it to the South China Sea (SCS). The detected submesoscale eddies are found to have a radius of $13 \pm 5$ $\mathrm{km}$ and an aspect ratio of $0.5 \pm 0.2$, with a notable predominance of cyclones. Further investigation reveals that the surface structure of these eddies displays a unique 'cat'seye' pattern and the eddies become more circular with increasing eddy radius. Submesoscale eddies can strongly regulate surface chlorophyll via horizontal advection while they have less coherent signatures in sea surface temperature. These findings may help to improve submesoscale parameterizations in Earth system models.
\end{abstract}

\title{
Plain Language Summary
}

Ubiquitous ocean eddies play a crucial role in the upper ocean dynamics. Using high-resolution satellite remote sensing data, we have developed an automatic method to detect small elliptical eddies in the SCS over a 10-year period. The results show that these 'submesoscale' eddies of the order of $10 \mathrm{~km}$ appear to have a unique 'cat's-eye' structure with significant effect on the surface tracer distribution. This study therefore improves our understanding of oceanic submesoscale dynamics and contributes to parameterizing the impact of submesoscale eddies in climate and ocean models.

\section{Introduction}

Submesoscale spiral eddies of the order of $10 \mathrm{~km}$ have been frequently observed in different regions over the world ocean since they were first seen in the sun-glitter from the Apollo Mission in 1968 (e.g., Munk et al., 2000; Shen and Evans, 2002; Buckingham et al., 2017). Although submesoscale eddies are believed to be important for upper ocean dynamics and biogeochemical processes (Haine and Marshall, 1998; Munk et al., 2000; McWilliams, 2010; Mahadevan, 2016), progress in characterizing 
and understanding them has been slow, because the resolutions of in-situ ocean measurements and satellite altimetry observations are typically too coarse to resolve these small-scale and short-lifetime eddies. One way to overcome this obstacle is to utilize other satellite remote sensing data, such as sea surface temperature (SST) and near-surface chlorophyll, which is available at high resolution and wide coverage (Munk et al., 2000; Liu et al., 2014; Buckingham et al., 2017). However, to our knowledge, no methods exist yet that are able to extract submesoscale spiral eddies from the remote sensing images in an automatic and systematic way. In this study, we first develop an automatic submesoscale eddy detection method and then apply it to the South China Sea (SCS), the largest marginal sea in the western Pacific that is rich in submesoscale eddies.

The SCS is characterized by varying seafloor topography, a seasonal upper ocean circulation, a complex upwelling-front system and active mesoscale eddies, which facilitate the generation of submesoscale phenomena (Wang et al., 2003; Hu and Wang, 2016; Lin et al., 2020). Although submesoscale eddies have been seen a few times in remote sensing data in the northern and western SCS (e.g., Su, 2004; Liu et al., 2014; Yu et al., 2018), the statistical properties of these eddies in the SCS (e.g., size, polarity and shape) have not been determined. In a seminar paper on spiral eddies, Munk et al. (2000) proposed that the surface structure of submesoscale spiral eddies can be described by an extension of the classical Stuart (1967) solution, which yields the wellknown 'cat's eye' configuration (Thomson, 1880; Fig. 1a). However, this cat's-eye surface structure proposed for submesoscale eddies is yet to be observationally confirmed and the key parameter in the Stuart solution to be determined. Automatic submesoscale eddy detection enables composite analyses of chlorophyll and SST anomalies associated with these eddies and as such is a useful tool for analyzing the surface structure of submesoscale eddies as well as their impact on surface tracer distributions.

This article is protected by copyright. All rights reserved. 


\section{Data}

The daily Moderate Resolution Imaging Spectroradiometer (MODIS) chlorophyll and SST data from the National Aeronautics and Space Administration (NASA) Ocean Colour project are analyzed in this study for a 10-year period from January 2006 to December 2015. Both the chlorophyll and SST data are level-2 products provided with a spatial resolution of $\sim 1 \mathrm{~km}$. Because of the log-normal distribution of chlorophyll concentration, we follow Chelton et al. (2011) and $\log _{10}$ transform the chlorophyll field before compositing chlorophyll anomalies associated with submesoscale eddies.

\section{Results}

\subsection{Statistical Features}

We first develop an automatic submesoscale eddy detection method based on the curvature of contours extracted from high-resolution chlorophyll data. The chlorophyll images are first processed to fill small blank patches due to clouds (Oram et al., 2008). The extracted chlorophyll contours are then broken into segments according to the contour curvature direction. The clustering segments that curl in the same direction are regarded as different parts of the same submesoscale eddy if they further satisfy a number of criteria. The type, edge and center of a submesoscale eddy are defined as the type, convex hull and geometric center of the segments of the eddy, respectively. A detailed description of the automatic submesoscale eddy detection method is provided in the Supporting Information (Fig. S1). For example, based on this method, two cyclonic submesoscale eddies are identified in the western SCS during the summer of 2012 (Fig. 1b) and an anticyclonic submesoscale eddy is detected in the eastern SCS during the winter of 2012 (Fig. 1c). Overall, about 5983 (4372) snapshots of cyclonic (anticyclonic) submesoscale eddies are identified in the entire SCS over the 10-year study period. The elevated number of cyclonic submesoscale eddies over their anticyclonic counterparts is consistent with the findings of previous theoretical and numerical studies that anticyclonic submesoscale eddies are subject to inertial 
instability while cyclonic submesoscale eddies are not (Munk et al., 2000; Shen and Evans, 2002; Dong et al., 2007; Hasegawa et al., 2009). Note that in weakly-stratified waters anticyclonic eddies are found to be more stable than cyclonic eddies (Buckingham et al. 2020). Submesoscale eddies in the SCS are frequently detected in the coastal regions (Fig. 1d), including the northern SCS shelf-slope region, both sides of the Luzon strait and the coastal waters off Vietnam, where submesoscale eddies have been reported before (e.g., Su, 2004; Zheng et al., 2008; Liu et al., 2014). In these boundary regions, enhanced along-slope velocity shear, strong coastal front instability and vortex stretching due to tidal flow over shallow waters are known to be able to generate submesoscale eddy activity (Munk et al., 2000; Gula et al., 2015; Li et al., 2020). A recent high-resolution modelling study by Lin et al. (2020) confirms that submesoscale processes are particularly active in these coastal regions of the SCS. Furthermore, the large chlorophyll gradients near the coast (Fig. S2a) facilitate identification of submesoscale eddies via our detection method which is based on chlorophyll contours. For both types of submesoscale eddies, they are more frequently detected in winter and summer while less in spring and autumn (Fig. S3), which is probably related to the strongly seasonally-varying upper ocean circulation in the SCS driven by the monsoon (Wang et al., 2003; Su, 2004; Liu et al., 2014).

Here we define the radius of a submesoscale eddy as the radius of a circle that has the same area as the eddy. Statistical analysis shows that the radii of submesoscale eddies in the SCS range from about $3 \mathrm{~km}$ to more than $30 \mathrm{~km}$, with a mean value of $14.2 \mathrm{~km}(13.4 \mathrm{~km})$ and a standard deviation of $5.2 \mathrm{~km}(4.5 \mathrm{~km})$ for cyclones (anticyclones) (Table 1; Fig. 2a). The eddy radii estimated in this study are comparable in magnitude to those estimated from various data in previous research (Liu et al., 2014; Xu et al., 2015; Yu et al., 2018). When it comes to characterizing eddy shape, one useful metric is eddy aspect ratio, which is defined as the ratio between the minor and major radius of the fitted ellipse. The probability density function of the aspect ratios of submesoscale eddies contains a skewed distribution (Fig. 2b), with an average of 0.48 (0.49) and a standard deviation of 0.18 (0.18) for cyclones (anticyclones) (Table 1). 
Interestingly, the eddy aspect ratio is found to be a function of the eddy radius, irrespective of the eddy polarity (Fig. 2c); the larger the submesoscale eddies, the more circular they are.

\subsection{Horizontal Structure}

The identified eddy edges are also used to investigate the horizontal structure of submesoscale eddies. We first create a rotated coordinate system for the eddies, where the coordinate center is defined as the center of each eddy, with the major (minor) axis of the eddy on the x-axis (y-axis) (Supporting Information; Fig. S4). After that, we project the edges of cyclonic and anticyclonic submesoscale eddies separately onto the rotated eddy coordinate (Figs. 3a, b and S5). The average edges of cyclonic and anticyclonic submesoscale eddies are found to be almost identical, revealing a nearly perfect 'cat's-eye' structure as shown in previous theoretical and numerical studies (Munk et al., 2000; Shen and Evans, 2002). We then compare the observed mean edges of submesoscale eddies with the Stuart solution $\psi=-U / k \cdot \log (\cosh (k y)-\alpha$. $\cos (k x)$ ), where $\mathrm{U}= \pm 0.3 \mathrm{~m} \mathrm{~s}^{-1}$ is the background shear flow, $k \approx 0.0003 \mathrm{~m}^{-1}$ is the ratio between $2 \pi$ and eddy length scale, and $\alpha$ is an unknown parameter between 0 and 1 that needs to be determined (following Munk et al., 2000). The Stuart solution yields parallel shear flows when $\alpha=0$ and concentrated point vortices as $\alpha$ approaching 1. By adjusting $\alpha$ to obtain a best fit of the Stuart solution to the observed eddies, both cyclonic and anticyclonic, we find $\alpha=0.6$ gives a good agreement. Our result therefore provides the first statistical observational evidence in support of the 'cat's-eye' horizontal structure proposed by Munk et al. (2000) for submesoscale eddies.

Given that the submesoscale eddy aspect ratio depends on eddy radius (Fig. 2c), the value of $\alpha$ in the Stuart solution may also vary with the radius of submesoscale eddies. To test this conjecture, we divide the identified eddies into five bins, at an interval of $5 \mathrm{~km}$ from $5 \mathrm{~km}$ to $30 \mathrm{~km}$, according to the eddy radius. Then, we average all the fitted ellipse edges of submesoscale eddies in each bin to estimate the best-fitting $\alpha$ for each bin. The value of $\alpha$ is indeed found to vary with the submesoscale eddy

This article is protected by copyright. All rights reserved. 
radius, increasing from over 0.4 to around 0.7 , with slightly smaller values for cyclones (Fig. 3c). Moreover, binning of $\alpha$ as a function of the radius of cyclonic (anticyclonic) submesoscale eddies displays a nearly linear relationship, with $\alpha=0.015 r+0.322$ $(\alpha=0.015 r+0.344)$ where $r$ is the radius of submesoscale eddies. The relationship between the eddy radius and $\alpha$ found in this study can be used to improve the Stuart solution to better describe the surface structure of submesocale eddies which may have implications for submesoscale eddy parameterizations.

\subsection{Composite chlorophyll and SST}

To examine the impact of submesoscale eddies on surface tracer distributions, the $\log _{10}$-transformed chlorophyll and SST data of the 10-year study period are first highpass filtered using a Gaussian filter (Ni et al., 2020) and then are projected and averaged onto the rotated submesoscale eddy coordinate (Supporting Information; Fig. S4). Note that the flank of an eddy with positive chlorophyll anomalies is taken as the positive yaxis. Fig. 4a (b) shows the resulting composite chlorophyll anomalies inside and around cyclonic (anticyclonic) submesoscale eddies detected in the SCS. On average, the magnitude of $\log _{10}$-transformed chlorophyll anomalies induced by submesoscale eddies is on the order of $\pm 0.1 \mathrm{mg} \mathrm{m}^{-3}$, which is comparable to the magnitude of seasonal variations of surface chlorophyll anomalies averaged over the SCS (Fig. S2b) but several times larger than that associated with mesoscale eddies (Chelton et al., 2011; Gaube at al., 2014; He at al., 2019). We also note that the composite chlorophyll anomalies indicate a 'cat's-eye' shape and display a distinct dipole pattern which consists of two rotational anomalies of opposite sign. Similar dipole structure has been seen in the composite maps of tracer anomalies (i.e., chlorophyll and SST) induced by mesoscale eddies, which is known to result from lateral eddy advection of background tracer gradients (Chelton et al., 2011; Hausmann and Czaja, 2012; Gaube et al., 2015). In regions of significant background chlorophyll gradient, the effect of horizontal eddy rotation is to advect high (low) chlorophyll concentration to the side of low (high) chlorophyll concentration and thereby result in positive (negative) chlorophyll 
anomalies. Indeed, the composite maps of Figs. $4 \mathrm{a}$ and $\mathrm{b}$ indicate the existence of distinct chlorophyll fronts at $y \approx 0$.

The composite SST anomalies associated with the identified cyclonic and anticyclonic submesoscale eddies are shown in Figs. 4c and d, respectively. One outstanding feature is that positive (negative) SST anomalies on the flanks of submesoscale eddies are collocated with negative (positive) chlorophyll anomalies, consistent with the fact that near the coast the chlorophyll concentration is higher while the SST is colder. Furthermore, the signatures of submesoscale eddies in the composite SST anomaly images tend to be more obscure when compared to chlorophyll. One possible explanation is that there exist various formation mechanisms for submesoscale eddies. For the mechanism of frontal instability, the pattern of chlorophyll anomalies is expected to be similar to that of SST anomalies (Munk et al., 2000; Klein and Lapeyre, 2009). For the mechanism of shear instability, however, a different picture occurs. For example, submesosocale eddies caused by flow-island interaction may occur in a relatively homogeneous temperature field (Fig. S1f; Yu et al., 2018), and as a result the imprint of submesoscale eddies in the SST anomalies are less pronounced. Previous research indeed found greater chlorophyll variance at submesoscales than SST (Mahadevan, 2016). This is why we choose chlorophyll rather than SST to identify subemesoscale eddies in our method. The difference between submesoscale eddy signatures in chlorophyll and SST maps also reflects the degree of conservativeness in their behaviour, which may need to be accounted for when parameterizing the effect of submesoscale eddies in the tracer equations.

\section{Conclusions}

In this work we have developed an automatic submesoscale spiral eddy identification method based on high-resolution chlorophyll data and then applied it to the SCS which is a marginal sea rich in submesoscale eddies. The detected submesoscale eddies in the SCS are found to have a radius of $13 \pm 5 \mathrm{~km}$ and an aspect ratio of $0.5 \pm 0.2$, with a notable predominance of cyclones. We have shown that the 
surface structure of submesoscale eddies displays the classical 'cat's-eye' pattern and further determined the key unknown parameter in the Stuart solution that describes the shape of the cat's-eye pattern. Submesoscale eddies are found to induce dipole surface chlorophyll and SST anomalies via horizontal advection of background chlorophyll and SST gradients.

The widespread existence of submesoscale eddies is believed to be important in tracer transport, energy cascade, re-stratification and biological processes in the upper ocean (Ubelmann and $\mathrm{Fu}, 2011$; McWilliams, 2010; Haine and Marshall, 1998; Mahadevan, 2016). However, the present global ocean and climate models have too coarse spatial resolutions to resolve submesoscale processes and as such would rely on parameterizing the effect of submesoscale eddies for the foreseeable future (e.g., FoxKemper et al., 2011). The submesoscale eddy structure and statistics found in this study may provide observation-based guidance for future development of submesoscale eddy parameterizations. For example, anisotropy in submesoscale eddy length scales, i.e., shorter length scale in the cross-front direction than along-front direction, implies anisotropic submesoscale eddy diffusivity if the parameterization scheme employs a mixing length approach.

The high-resolution Surface Water and Ocean Topography (SWOT) satellite altimeter is scheduled to launch in 2021 (Qiu et al., 2017), which aims at resolving sea level variability at submesoscales. Combining the chlorophyll-based submesoscale eddy detection method developed in this study with SWOT-derived submesoscale sea level anomalies should have potential to further improve our understanding of the surface pattern, dynamics and impact of submesoscale eddies. Nevertheless, in addition to satellite remote sensing, we still need in-situ observing technologies with highenough spatiotemporal resolution to reveal the three-dimensional structure of these eddies.

This article is protected by copyright. All rights reserved. 


\section{Acknowledgments}

X.Z. acknowledges support by a Royal Society International Exchanges Award (IEC/NSFC/170007). C.W. is supported by the Climate Linked Atlantic Sector Science (CLASS) project, which is itself supported by NERC National Capability funding (NE/R015953/1). C.C. acknowledges support by the National Natural Science Foundation of China (42076018). D.C. is supported by the National Natural Science Foundation of China (41730535). Q.N. thanks Guihua Wang, Xuemin Jiang, Pengfei Tuo, Sheng Lin and Zhibin Yang for their helpful discussions. The chlorophyll and SST data are available at https://oceandata.sci.gsfc.nasa.gov/.

\section{References}

1. Buckingham, C. E., Gula, J., \& Carton, X. (2020). The role of curvature in modifying frontal instabilities, part 2. Journal of Physical Oceanography, 1-67.

2. Buckingham, C. E., Khaleel, Z., Lazar, A., Martin, A. P., Allen, J. T., Garabato, A. C., Thompson, A. F., \& Vic, C. (2017). Testing Munk's hypothesis for submesoscale eddy generation using observations in the North Atlantic. Journal of Geophysical Research, 122(8), 6725-6745.

3. Chelton, D. B., Schlax, M. G., \& Samelson, R. M. (2011). Global observations of nonlinear mesoscale eddies. Progress in Oceanography, 91(2), 167-216.

4. Dong, C., Mcwilliams, J. C., \& Shchepetkin, A. F. (2007). Island Wakes in Deep Water. Journal of Physical Oceanography, 37(4), 962-981.

5. Fox-Kemper, B., Danabasoglu, G., Ferrari, R., Griffies, S. M., Hallberg, R. W., Holland, M. M., Maltrud, M. E., Peacock, S., \& Samuels, B. L. (2011). Parameterization of mixed layer eddies. III: Implementation and impact in global ocean climate simulations. Ocean Modelling, 39, 61-78.

This article is protected by copyright. All rights reserved. 
6. Gaube, P., Chelton, D. B., Samelson, R. M., Schlax, M. G., \& O’Neill, L. W. (2015). Satellite observations of mesoscale eddy-induced Ekman pumping. Journal of Physical Oceanography, 45(1), 104-132.

7. Gaube, P., Mcgillicuddy, D. J., Chelton, D. B., Behrenfeld, M. J., \& Strutton, P. G. (2014). Regional variations in the influence of mesoscale eddies on near-surface chlorophyll. Journal of Geophysical Research, 119(12), 8195-8220.

8. Gula, J., Molemaker, M. J., \& Mcwilliams, J. C. (2015). Topographic vorticity generation, submesoscale instability and vortex street formation in the Gulf Stream. Geophysical Research Letters, 42(10), 4054-4062.

9. Haine, T. W. N., \& Marshall, J. (1998). Gravitational, symmetric, and baroclinic instability of the ocean mixed layer. Journal of Physical Oceanography, 28(4), 634658.

10. Hasegawa, D., Lewis, M. R., \& Gangopadhyay, A. (2009). How islands cause phytoplankton to bloom in their wakes. Geophysical Research Letters, 36(20), L20605.

11. Hausmann, U., \& Czaja, A. (2012). The observed signature of mesoscale eddies in sea surface temperature and the associated heat transport. Deep Sea Research Part I, 70, 60-72.

12. He, Q., Zhan, H., Xu, J., Cai, S., Zhan, W., Zhou, L., \& Zha, G. (2019). Eddyinduced chlorophyll anomalies in the western South China Sea. Journal of Geophysical Research, 124, 1-20.

13. Hu, J., \& Wang, X. H. (2016). Progress on upwelling studies in the China seas. Reviews of Geophysics, 54(3), 653-673.

14. Thomson, W. (1880) On a disturbing infinity in Lord Rayleigh's solution for waves 
in a plane vortex stratum. Nature, 23, 45-46.

15. Klein, P., \& Lapeyre, G. (2009). The oceanic vertical pump induced by mesoscale and submesoscale turbulence. Annual Review of Marine Science, 1(1), 351-375.

16. Li, G., He, Y., Liu, G., Zhang, Y., Hu, C., \& Perrie, W. (2020). Multi-sensor observations of submesoscale eddies in coastal regions. Remote Sensing, 12(4): 711.

17. Lin, H., Liu, Z., Hu, J., Menemenlis, D., \& Huang, Y. (2020). Characterizing mesoto submesoscale features in the South China Sea. Progress in Oceanography, 118, 102420.

18. Liu, F., Tang, S., \& Chen, C. (2014). Satellite observations of the small-scale cyclonic eddies in the western South China Sea. Biogeosciences, 12(2), 299-305.

19. Mahadevan, A. (2016). The Impact of Submesoscale Physics on Primary Productivity of Plankton. Annual Review of Marine Science, 8(1), 161-184.

20. McWilliams, J. C. (2010). A perspective on submesoscale geophysical turbulence. IUTAM Symposium on Turbulence in the Atmosphere and Oceans, 131-141.

21. Munk, W., Armi, L., Fischer, K. W., \& Zachariasen, F. (2000). Spirals on the sea. Proceedings of The Royal Society A: Mathematical, Physical and Engineering Sciences, 456(1997), 1217-1280.

22. Ni, Q., Zhai, X., Wang, G., \& Marshall, D. P. (2020). Random movement of mesoscale eddies in the global ocean. Journal of Physical Oceanography, 50(8), 2341-2357.

23. Oram, J. J., Mcwilliams, J. C., \& Stolzenbach, K. D. (2008). Gradient-based edge detection and feature classification of sea-surface images of the Southern California Bight. Remote Sensing of Environment, 112(5), 2397-2415.

This article is protected by copyright. All rights reserved. 
24. Qiu, B., Nakano, T., Chen, S., \& Klein, P. (2017). Submesoscale transition from geostrophic flows to internal waves in the northwestern Pacific upper ocean. Nature Communications, 8, 14055.

25. Shen, C. Y., \& Evans, T. E. (2002). Inertial instability and sea spirals. Geophysical Research Letters, 29(23), 39-1-39-4.

26. Stuart, J. T. (1967). On finite amplitude oscillations in laminar mixing layers. Journal of Fluid Mechanics, 29, 417-440.

27. Su, J. (2004). Overview of the South China Sea circulation and its influence on the coastal physical oceanography outside the Pearl River estuary. Continental Shelf Research, 24(16), 1745-1760.

28. Ubelmann, C., \& Fu, L. (2011). Cyclonic eddies formed at the Pacific tropical instability wave fronts. Journal of Geophysical Research, 116, C12021.

29. Wang, G., Su, J., \& Chu, P. C. (2003). Mesoscale eddies in the South China Sea observed with altimeter data. Geophysical Research Letters, 30(21), 2121.

30. Xu, G., Yang, J., Dong, C., Chen, D., \& Wang, J. (2015). Statistical study of submesoscale eddies identified from synthetic aperture radar images in the Luzon Strait and adjacent seas. Journal of remote sensing, 36(18), 4621-4631.

31. Yu, J., Zheng, Q., Jing, Z., Qi, Y., Zhang, S., \& Xie, L. (2018). Satellite observations of sub-mesoscale vortex trains in the western boundary of the South China Sea. Journal of Marine Systems, 183, 56-62.

32. Zheng, Q., Lin, H., Meng, J., Hu, X., Song, Y. T., Zhang, Y., \& Li, C. (2008). Submesoscale ocean vortex trains in the Luzon Strait. Journal of Geophysical Research, 113, C04032.

This article is protected by copyright. All rights reserved. 


\section{Table}

Table 1. Statistical features of submesoscale eddies detected in the South China Sea from 2006 to 2015

\begin{tabular}{ccccc}
\hline \multirow{2}{*}{ Polarity } & \multicolumn{2}{c}{$\mathrm{r}(\mathrm{km})$} & \multicolumn{2}{c}{$r_{\text {min }} / r_{\text {maj }}$} \\
\cline { 2 - 5 } & Mean & STD & Mean & STD \\
\hline Cyclonic & 14.2 & 5.2 & 0.48 & 0.18 \\
Anticyclonic & 13.4 & 4.5 & 0.49 & 0.18 \\
\hline
\end{tabular}

This article is protected by copyright. All rights reserved. 


\section{Figures}
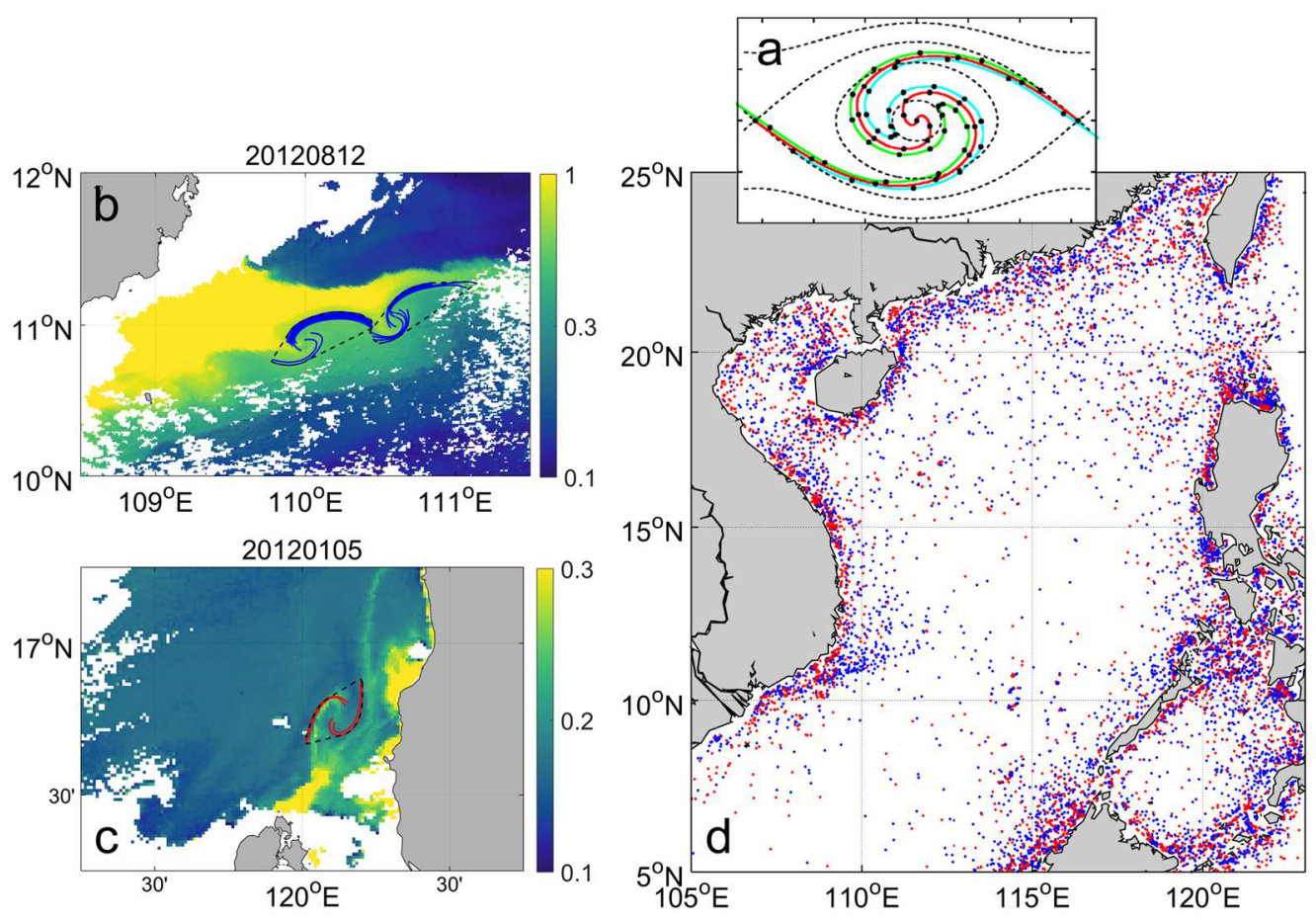

Figure 1. (a) Particle distribution (black dots and colour curves) in a Stuart spiral eddy (black dashed contour) that shows a 'cat's-eye' pattern. Adapted from Munk et al. (2000). (b) One-day snapshot of cyclonic submesoscale eddies (blue curves) identified from high-resolution chlorophyll data (colour shading; $\mathrm{mg} \mathrm{m}^{-3}$ ). The eddy edges are denoted by black dashed curves. (c) Same as Fig. $1 \mathrm{~b}$ but for an anticyclonic submesoscale eddy (red curves). (d) Distributions of cyclonic (blue dots) and anticyclonic (red dots) submesoscale eddies identified in the South China Sea (SCS) from 2006 to 2015 .

This article is protected by copyright. All rights reserved. 


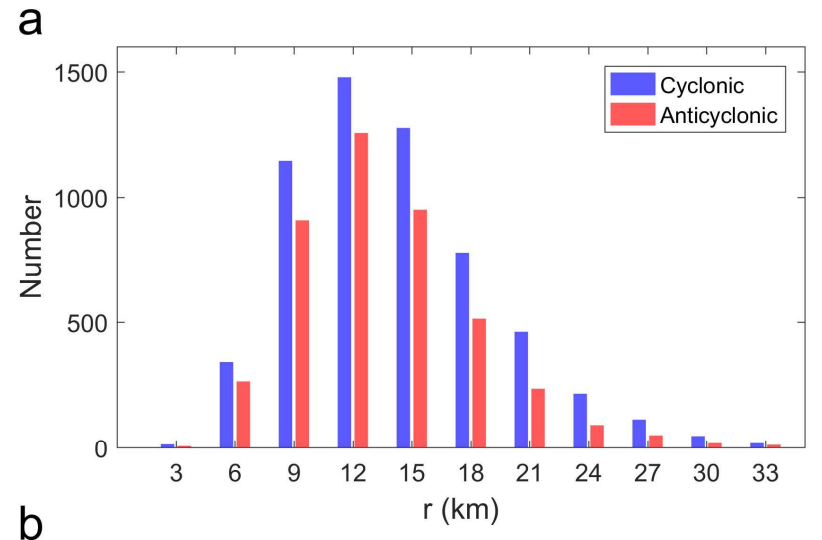

b
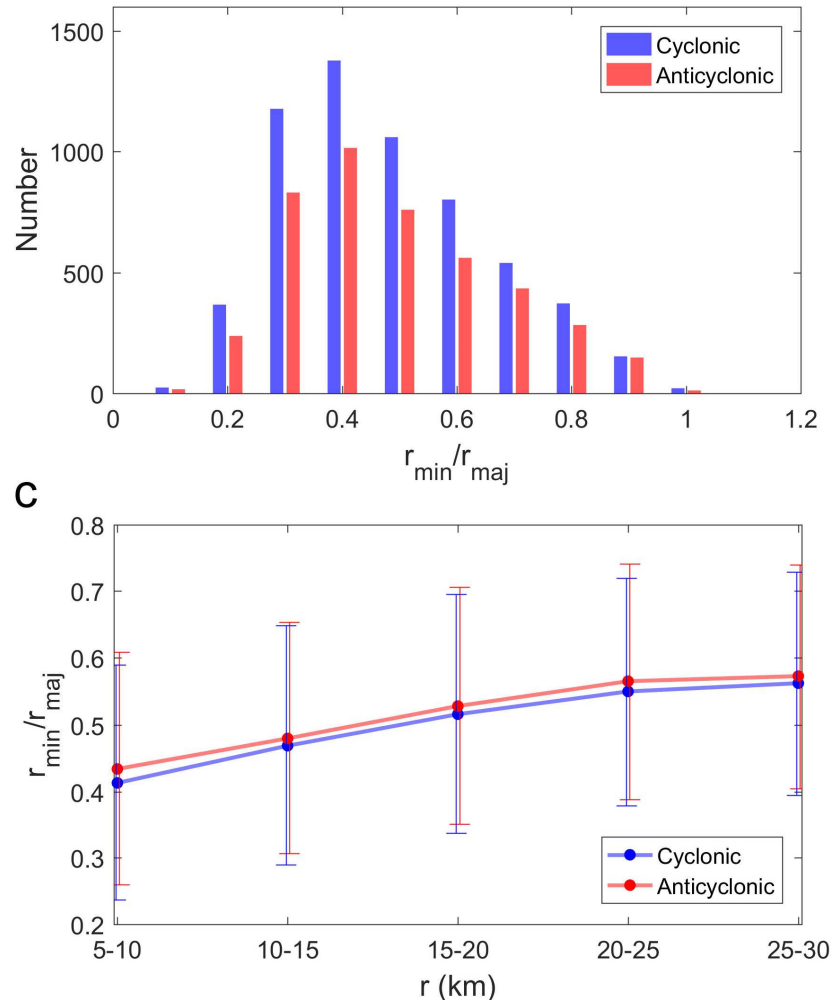

Figure 2. (a) Histogram of the radius of submesoscale eddies in the SCS. (b) Same as Fig. 2a but for the eddy aspect ratio that is defined as the ratio between the minor and major radius of a submesoscale eddy. (c) Variations of eddy aspect ratio with eddy radius (averaged in an eddy-radius bin of $5 \mathrm{~km}$ ). Vertical lines denote one standard deviation.

This article is protected by copyright. All rights reserved. 

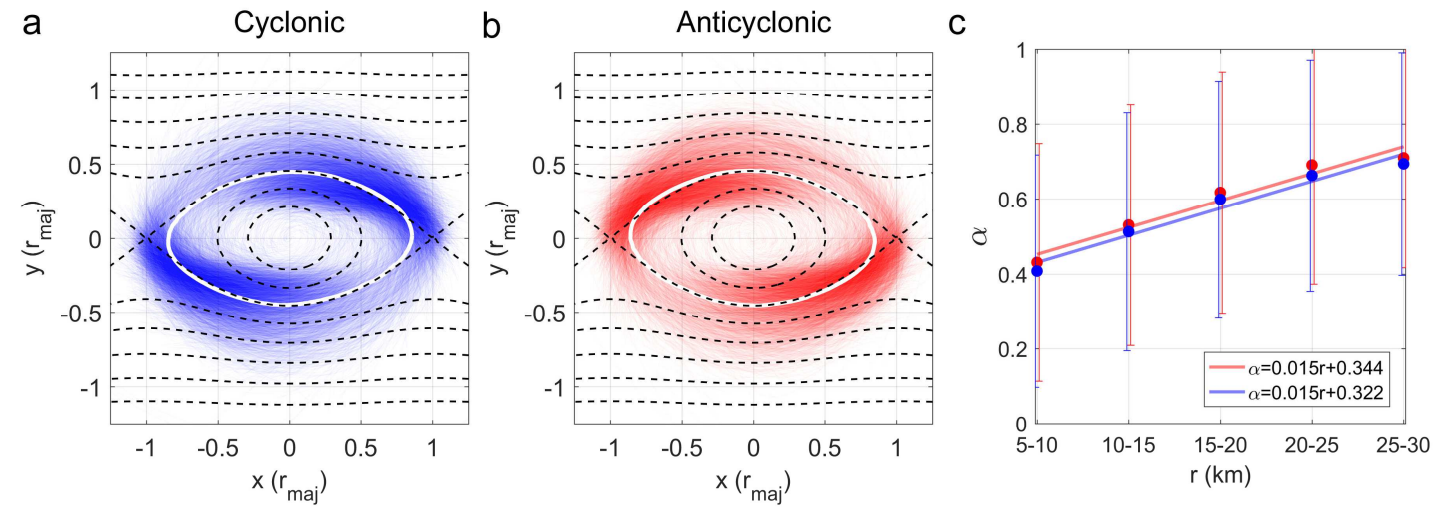

Figure 3. Horizontal structure of submesoscale eddies in the SCS. (a) Edges of cyclonic eddies (blue curves) and their average (white curve) on a rotated submesoscale eddy coordinate system (Supporting Information). Black dashed contours are the horizontally normalized streamfunction contours derived from the Stuart solution $\psi=$ $-U / k \cdot \log (\cosh (k y)-\alpha \cdot \cos (k x))$, where $U= \pm 0.3 \mathrm{~m} \mathrm{~s}^{-1}, k \approx 0.0003 \mathrm{~m}^{-1}$, and $\alpha=0.6$. (b) Same as Fig. 3a but for anticyclonic eddies (red curves). (c) Values of $\alpha$ as a function of the radius of cyclonic (blue dots) and anticyclonic (red dots) submesoscale eddies and the corresponding linear fitting results (lines).

This article is protected by copyright. All rights reserved. 


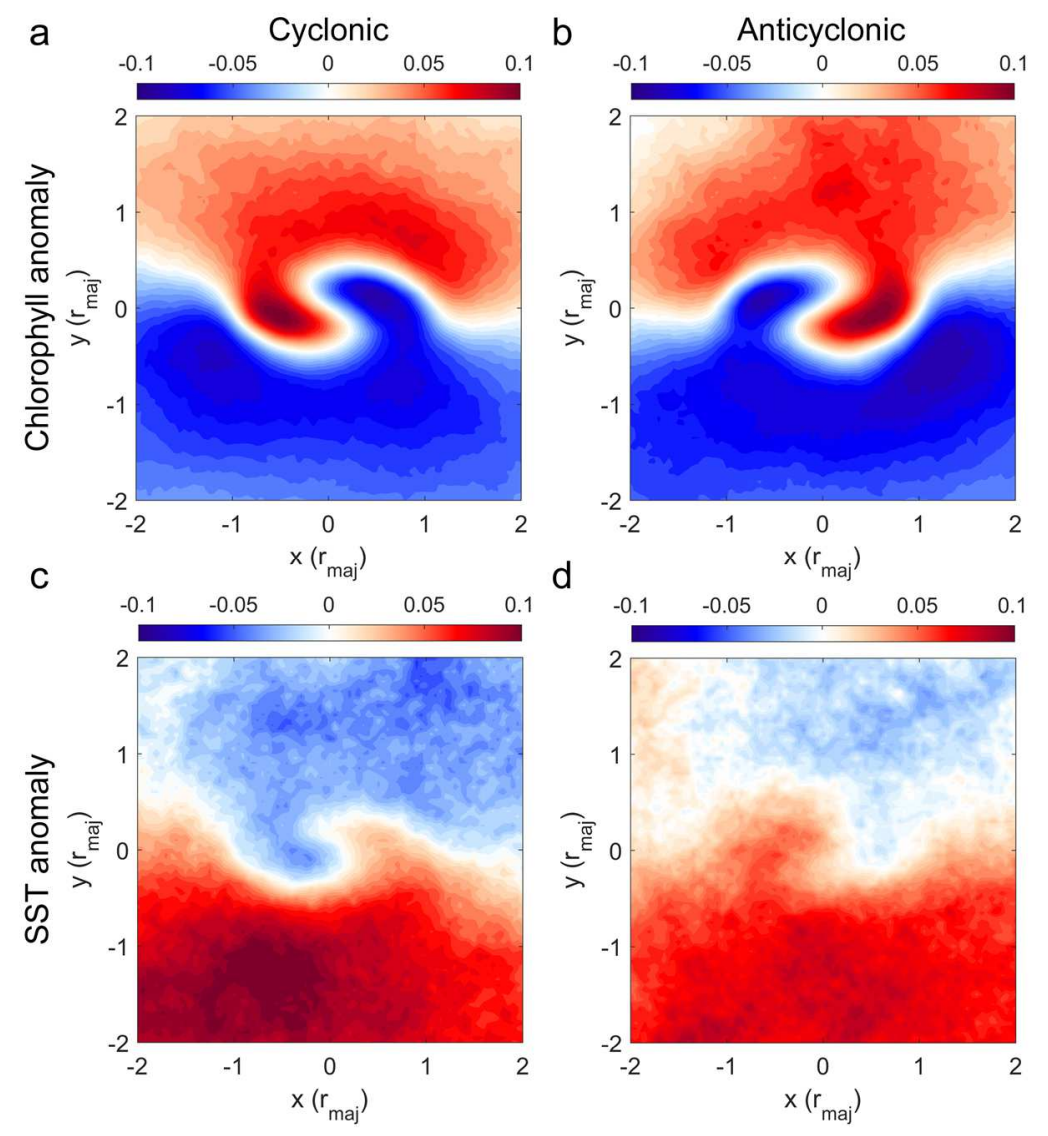

Figure 4. $(\mathrm{a}, \mathrm{b})$ Composite $\log _{10}$-transformed chlorophyll anomalies $\left(\mathrm{mg} \mathrm{m}^{-3}\right)$ on the rotated submesoscale eddy coordinate. (c, d) Same as Fig. 4a, b but for SST anomalies $\left({ }^{\circ} \mathrm{C}\right)$.

This article is protected by copyright. All rights reserved. 


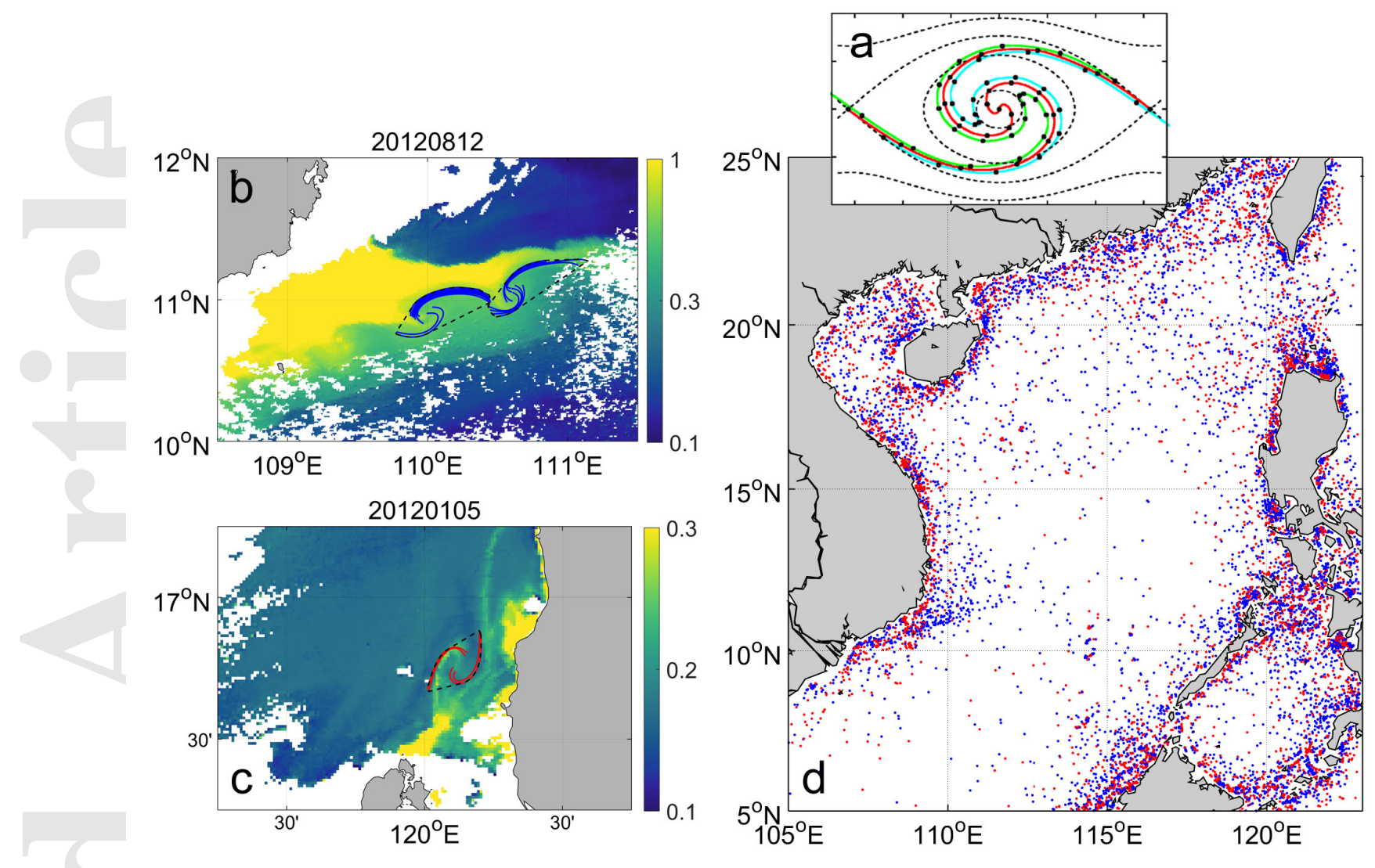

This article is protected by copyright. All rights reserved. 

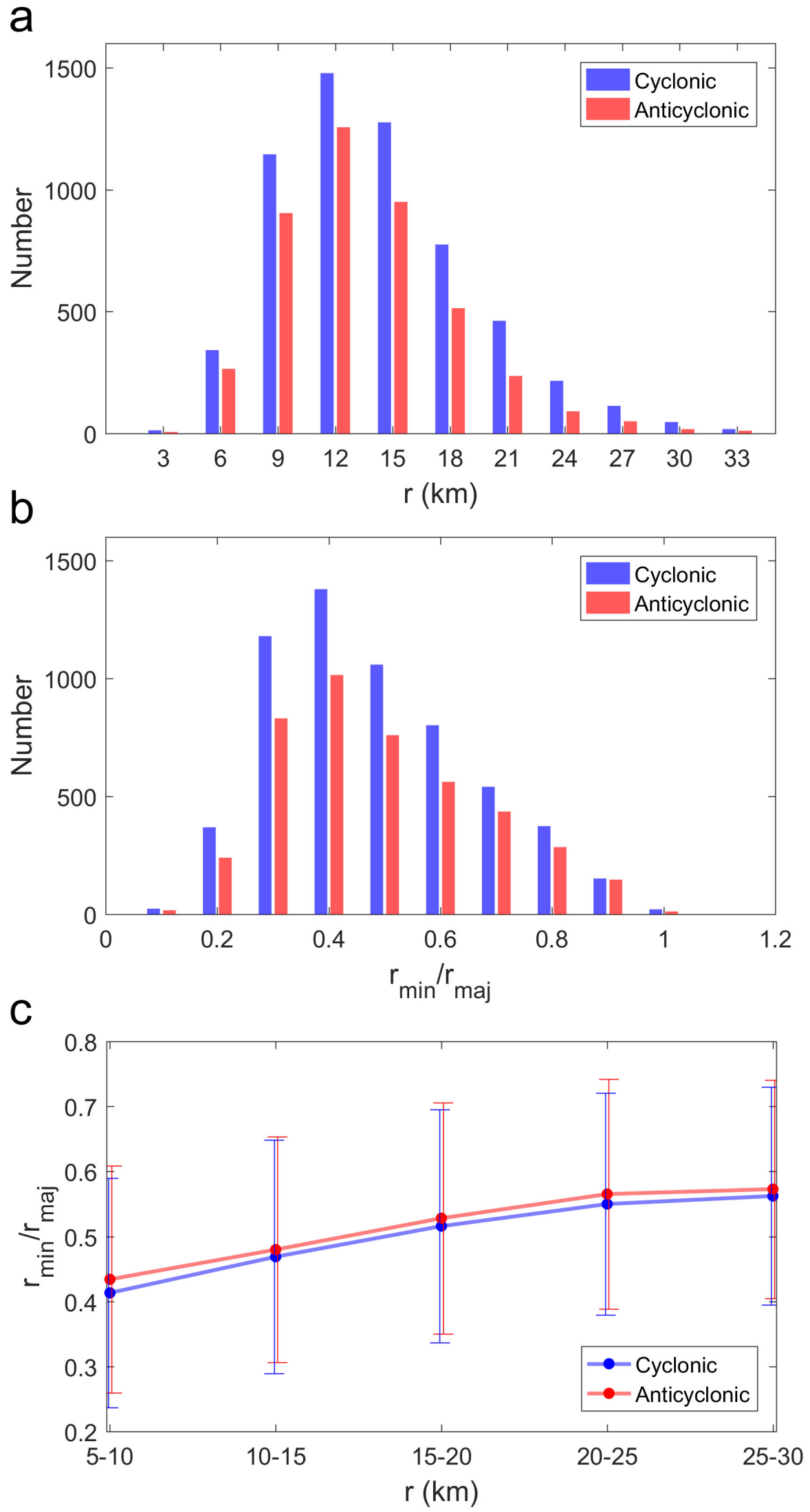

This article is protected by copyright. All rights reserved. 

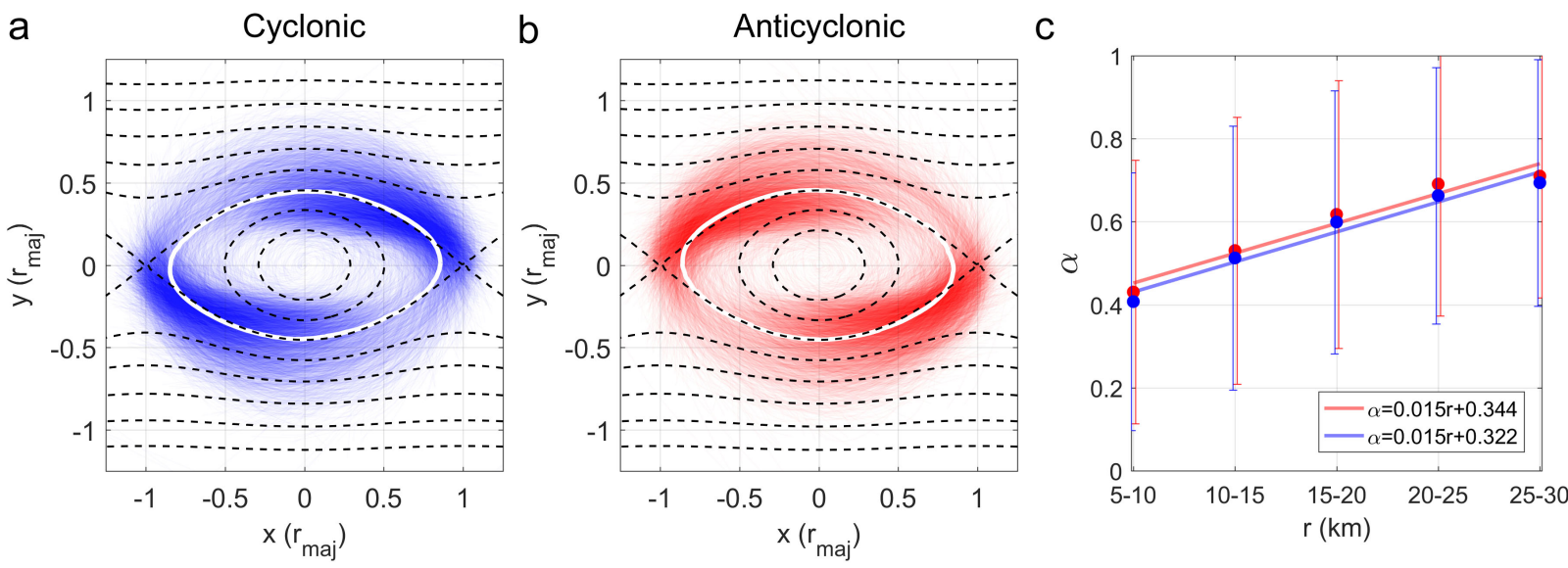

This article is protected by copyright. All rights reserved. 

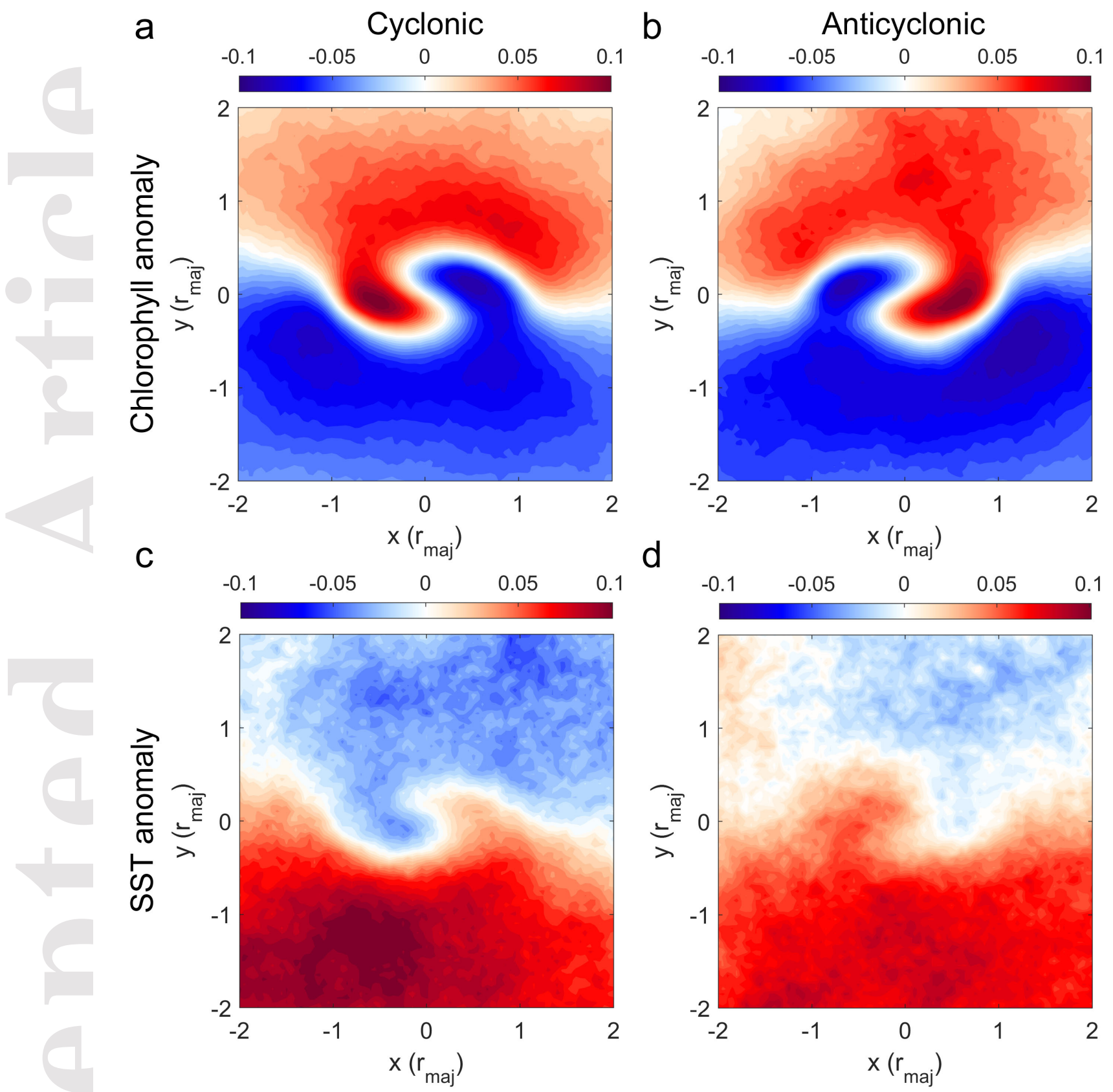

This article is protected by copyright. All rights reserved. 